\title{
Jean Wauquelin. De Mons à la cour de Bourgogne, publié sous la direction de Marie-Claude de Crécy
}

\section{Barbara Ferrari}

\section{(2) OpenEdition}

\section{Journals}

Édition électronique

URL : https://journals.openedition.org/studifrancesi/26008

DOI : 10.4000/studifrancesi.26008

ISSN : 2427-5856

Éditeur

Rosenberg \& Sellier

\section{Édition imprimée}

Date de publication : 1 avril 2007

Pagination : 155-156

ISSN : 0039-2944

\section{Référence électronique}

Barbara Ferrari, « Jean Wauquelin. De Mons à la cour de Bourgogne, publié sous la direction de Marie-

Claude de Crécy », Studi Francesi [En ligne], 151 (LI | I) | 2007, mis en ligne le 30 novembre 2015, consulté le 23 novembre 2021. URL : http://journals.openedition.org/studifrancesi/26008; DOI :

https://doi.org/10.4000/studifrancesi.26008

Ce document a été généré automatiquement le 23 novembre 2021.

\section{(c) (i) $\odot$}

Studi Francesi è distribuita con Licenza Creative Commons Attribuzione - Non commerciale - Non opere derivate 4.0 Internazionale. 


\title{
Jean Wauquelin. De Mons à la cour de Bourgogne, publié sous la direction de Marie-Claude de Crécy
}

\author{
Barbara Ferrari
}

\section{RÉFÉRENCE}

Jean Wauquelin. De Mons à la cour de Bourgogne, publié sous la direction de Marie-Claude DE CRÉCY avec la collaboration de Gabriella PARUSSA et de Sandrine HÉRICHÉ-PRADEAU, Turnhout, Brepols («Burgundica», XI), 2006, pp. 318.

1 L'intérêt pour Jean Wauquelin, dont l'activité multiforme liée à la production de livres, à la traduction, à la compilation et à la mise en prose de récits en vers s'est déployée pendant le deuxième quart du $\mathrm{xv}^{\mathrm{e}}$ siècle entre son atelier de Mons et la cour des ducs de Bourgogne, n'a cessé de s'accroitre ces dernières années. De nouvelles éditions de ses œuvres, inédites ou accessibles uniquement dans des publications de la fin du XIX siècle, ont vu le jour ou sont en préparation: après Les faicts et conquestes d'Alexandre le Grand, publiés en 2000 par Sandrine Hériché (TLF 527, SF 138, pp. 672-673), Marie-Claude de Crécy a procuré, en 2002, l'édition de la mise en prose de La Belle Hélène de Constantinople (TLF 547, SF 140, pp. 421-422); sont en outre annoncées: la traduction de l'Historia regum Britannie de Geoffroy de Monmouth, éditée par Géraldine Veysseyre (sous presse chez Droz), Girart de Roussillon (en préparation par Marie-Claude de Crécy) et une nouvelle édition de la mise en prose de la Manequine (en préparation par Maria Colombo Timelli). Parmi les études, il faut rappeler au moins les importantes contributions réunies dans le volume Les Chroniques de Hainaut, ou les ambitions d'un prince bourguignon (KBR-Brepols 2000, SF 145, pp. 135-136), avec la publication de plusieurs documents inédits. Il était temps donc, comme le souligne Marie-Claude de Crécy dans l'Introduction (pp. 1-4), de réunir les chercheurs qui travaillent sur Wauquelin pour faire le point sur l'état des connaissances et «ouvrir des perspectives, 
creuser les routes nouvelles que pourraient emprunter de futures recherches» (p. 1). Pour répondre à cette double attente, les spécialistes qui ont participé au Colloque organisé à Tours en septembre 2004, dont ce volume recueille les actes, ont abordé l'œuvre et l'activité de Wauquelin de différents points de vue: linguistique, littéraire, iconographique.

2 L'examen des procédés mis en acte par Wauquelin traducteur font l'objet des trois premières contributions: Gilles ROUSSINEAU, J. W. et l'auteur de Perceforest traducteurs de l'Historia regum Britannie de Geoffroy de Monmouth (pp. 5-23); Outi MERISAlO, J. W., traducteur de Gilles de Rome (pp. 25-31); Claude THIRY et Tania VAN HEMELRYCK, La langue de J. W. L'exemple des Chroniques de Hainaut (livre iii) (pp. 33-42). L'absence d'éditions critiques des modèles latins comprenant au moins une sélection des variantes constitue une limite sérieuse pour ce genre d'analyses dont il aurait fallu peut-être tenir compte davantage; ceci dit, la comparaison entre la technique de Wauquelin et celle d'autres traducteurs qui ont 'translaté' les mêmes textes met en lumière la fidelité et l'exactitude des traductions du clerc montois, qui garde toutefois une certaine marge de liberté; celle-ci se manifeste d'une part dans le refus de la traduction mot à mot et de l'emploi excessif de calques, d'autre part dans la prédilection pour les procédés d'amplification tels que gloses et binômes. Dans la traduction du De regimine principum de Gilles de Rome, l'alternance entre la recherche d'un vocabulaire roman et l'emploi de latinismes semble indiquer, selon Outi Merisalo, le manque d'une révision définitive; cependant, Claude Thiry et Tania Van Hemelryck nous invitent à rester sur nos gardes. Leur enquête, centrée sur le vocabulaire des Chroniques de Hainaut, montre en fait qu'un latinisme peut être absent de l'original et qu'une glose, binôme ou introduction métatextuelle, peut déjà se trouver dans la source latine. En relevant la présence dans le texte de Wauquelin de termes qui, après leur première apparition dans des traductions de la moitié du XIV siècle, semblaient avoir été abandonnés pour ne réapparaitre qu'au $\mathrm{xVI}^{\mathrm{e}}$ siècle, les deux auteurs avancent l'hypothèse intéressante de l'existence d'une «langue des traducteurs, vecteurs de diffusion lexicale» (p. 38) dont le ms. KBR 9242-44 des Chroniques constituerait un témoignage. Sur le versant linguistique toujours, la contribution de Géraldine VEYSSEYRE (L'itération lexicale dans la prose de J. W.: outil de traduction ou procédé ornemental?, pp. 43-68), entièrement consacrée aux séries de (pseudo-)synonymes, élargit le corpus aux compilations et aux mises en prose rédigées par Wauquelin; «nette prépondérance de la valeur ornementale par rapport à l'emploi explicatif, goût permanent pour les itérations équilibrées au plan rythmique, refus des effets voyants» (p. 62) sont quelques-unes des constantes mises en relief par son enquête, qui présente aussi un intérêt méthodologique plus général. Cette observation convient également à l'essai de Claude Roussel centré sur Les rubriques de La Belle Hélène de Costantinople (pp. 201-212), mais qui réfléchit aussi sur la fonction des titres de chapitre dans les réécritures de la fin du Moyen Âge: «balisage narratif [laissant] entrevoir de nouvelles exigences d'accès aux textes, privilégiant une approche plus rapide, plus sélective» (p. 212). Wauquelin dérimeur fait encore l'objet de l'étude de Maria colombo timelli (La rhétorique épistolaire dans deux mises en prose de J. W.: La Manequine et La Belle Hélène de Constantinople, pp. 69-88). L'apport de son enquête va au-delà de l'analyse stylistique et linguistique de la prose épistolaire du remanieur puisqu'elle fournit des éléments utiles à l'établissement de la chronologie relative des deux mises en prose (antériorité de La Manequine par rapport à La Belle Hélène, dediée à Philippe le Bon en 1448). L'étude de la langue peut aider aussi à mieux comprendre 
l'organisation du travail dans l'atelier de Wauquelin; c'est ce qui ressort de la contribution de Gabriella PARUSSA et Richard TRASCHLER (La scripta de Jacotin du Bois, un copiste dans l'atelier de J. W., pp. 185-200), qui tracent le 'profil orthographique' de l'un des scribes qui collaboraient habituellement avec Wauquelin pour mieux définir le rôle de Jacotin dans l'élaboration des trois manuscrits bruxellois des Chroniques de Hainaut et du manuscrit parisien de l'Alexandre.

3 L'approche littéraire caractérise une deuxième série d'études thématiques opérant une lecture croisée entre les ouvrages du remanieur bourguignon et leurs sources. Sandrine HÉRICHÉ-PRADEAU (Girart de Roussillon: la stratégie hagiographique d'une compilation, p. 89-109) met en lumière l'«amplification du versant hagiographique» (p. 90) de la légende de Girart, opérée par Wauquelin au niveau formel, thématique et lexical, par rapport à sa source principale, la version anonyme du XIV ${ }^{\mathrm{e}}$ siècle en couplets d'alexandrins. Rosalind BROWN-GRANT (Ré-écritures de récits d'inceste: J. W. et la légende de la "fille aux mains coupées", pp. 111-122) analyse le traitement du motif de l'inceste dans La Manekine de Philippe de Remi, Le Roman du Comte d'Anjou de Jehan Maillart et la mise en prose de Wauquelin. À son avis, cette dernière prend ses distances de la version de Philippe de Remi «pour s'insérer de façon assez originale dans le courant anti-courtois lancé par Jehan Maillart» (pp. 117-118). R. Brown-Grant y décèle l'influence des 'miroirs des princes', tels le De regimine principum de Gilles de Rome, qui permet à Wauquelin de traiter le sujet de l'inceste selon un axe politico-moral. Les Faicts et Conquestes d'Alexandre le Grand font l'objet de trois réflexions: Laurence HARF-LANCNER (Les portes $d u$ rêve chez J. W.: les songes dans le Roman d'Alexandre, pp. 123-135) analyse la nature et la fonction narrative et structurelle des rêves dans la compilation de Wauquelin en montrant aussi le rôle que l'écrivain leur attribue dans la glorification du héros. Cynthia SYOEN (Le merveilleux périple d'Alexandre: une vision pré-chrétienne et apologétique, pp. 137-155) interprète l'exploration d'Alexandre comme un voyage initiatique: non seulement «recherche sur les secrets du monde», mais aussi «quête de lui-même vers laquelle le dirigent les instances supérieures» (p. 137). Cette épreuve représente alors dans la compilation de Wauquelin «une forme d'élection divine et un chemin de rédemption qui illustre la miséricorde de Dieu» (p. 155). Enfin, Maud PÉREZ-SIMON (Les proverbes: une stratégie littéraire de Miroir de Prince. J. W. et les sources des Faicts et Conquestes d'Alexandre le Grand, pp. 157-170) étudie les transformations subies par le discours sentencieux qui apparaît d'abord comme 'parole guerrière' insérée dans un registre épique, mais que Wauquelin réussit ensuite à adapter à un contexte idéologique chevaleresque et courtois. L'image de l'orient, thématique fondamentale dans la littérature bourguignonne du XV siècle, est analysée par David WRISLEY (L'Orient de J. W., pp. 171-184), qui prend en compte l'ensemble de la production épicoromanesque de Wauquelin. Le critique reconnaît une progression dans la représentation de l'Orient: le procédé du remaniement chez Wauquelin ne serait pas lié uniquement à la propagande pour la croisade, mais aussi «à la construction d'une identité propre au duc et aux nobles de sa cour» (p. 183).

Les trois dernières contributions apportent des informations précieuses pour une meilleure connaissance et appréciation de Wauquelin iconographe, qui a souvent participé à la conception de l'illustration des manuscrits de ses œuvres à côté d'artistes tels que Dreux Jehan, enlumineur officiel de Philippe le Bon (Chrystèle BLONDEAU, J. W. et l'illustration de ses textes. Les exemples des Faicts et Conquestes d'Alexandre le Grand (Paris, BnF, ms. fr. 9342) et du Girart de Roussillon (Vienne, ÖNB, ms. 2549), pp. 213-224; 
Marie-Hélène TESNIÈRE, Les dieux antiques dans les Chroniques de Hainaut de J. W., pp. 225-243; Anne VAN BUREN, Les portraits de W. et son rôle d'iconographe: l'apport du costume, pp. 245-255).

5 La bibliographie finale (J. W., ses œuvres et les manuscrits qui les conservent, pp. 257-295), préparée par Géraldine Veysseyre, compte 256 items; si elle répond à l'objectif primaire de dresser un bilan exhaustif des études concernant Wauquelin, elle constitue aussi un outil indispensable pour toute nouvelle recherche. Je me permets de signaler quelques petits compléments: ajouter aux c.r. de l'édition Hériché des Faits et conquestes celui de Giovanni Palumbo, Medioevo Romanzo, XXIV (2000), pp. 316-317; ajouter aux études: Olöf Brattö, La Belle Hélène de Constantinople. Conte d'aventures du XV siècle. I. Introduction. La version d'Arras, Thèse, Stockolm 1959 (qui contient aussi une courte analyse de la mise en prose anonyme et de celle de Wauquelin et publie l'épisode de la prise de Jérusalem dans toutes les versions); Rudolf Ruths, Die französischen Fassungen des Roman de la Belle Helaine, Greifswald 1897 (étude des rapports de filiation entre les trois versions, le poème et les deux proses, analyse synoptique des textes); par ailleurs, le n. 27, p. 265, de Maria Colombo Timelli, ne concerne pas la Belle Hélène de Wauquelin mais la version anonyme.

Un dossier de dix-huit illustrations en noir et blanc (pp. 296-318) complète le volume.

7 Tant pour la variété des aspects traités que pour la richesse des contributions, et surtout pour la précieuse bibliographie, cet ouvrage constituera dorénavant un point de départ incontournable pour les recherches à venir. 